\title{
Controllable Effective Threshold Based Fusion Coverage Algorithm in Mobile Sensor Networks
}

\author{
Yong Lu $(\mathbb{D})$ and Na Sun \\ School of Information Engineering, Minzu University of China, Beijing 100081, China \\ Correspondence should be addressed to Yong Lu; amycun@126.com
}

Received 25 December 2017; Accepted 5 March 2018; Published 4 April 2018

Academic Editor: Jaime Lloret

Copyright (C) 2018 Yong Lu and Na Sun. This is an open access article distributed under the Creative Commons Attribution License, which permits unrestricted use, distribution, and reproduction in any medium, provided the original work is properly cited.

\begin{abstract}
The coverage quality and network lifetime are two key parameters in the research of sensor networks. The coverage quality shows direct influences on the network lifetime. Meanwhile, it is influenced by many other factors such as physical parameters and environmental parameters. To reveal the connection between the coverage quality and the parameters of target node concerned, a fusion coverage algorithm with controllable effective threshold is proposed based on the sensing probability model. We give the model for the membership function of coverage intensity as well as the prediction model for the fusion operator. The range for the effective threshold is presented according to the membership function model. Meanwhile, the maximum of the effective coverage intensity for the target nodes within the monitoring area is derived. The derivation of the maximal fusion coverage intensity is elaborated utilizing a processing function on the distances from the target node to the ones in the sensor node set. Furthermore, we investigate different network properties within the monitoring area such as network coverage quality, the dynamic change of parameters, and the network lifetime, based on the probability theory and the geometric theory. Finally, we present numerical simulations to verify the performances of our algorithm. It is shown under different settings that, compared with the demand coverage quality, the proposed algorithm could improve the network coverage quality by $15.66 \%$ on average. The simulation experiment results show that our proposed algorithm has an average improvement by $10.12 \%$ and $13.23 \%$ in terms of the performances on network coverage quality and network lifetime, respectively. The research results are enlightening to the edge coverage and nonlinear coverage problems within the monitoring area.
\end{abstract}

\section{Introduction}

The wireless sensor networks (WSNs) are constructed by thousands of sensor nodes in a self-organized and multihop way. The WSN accomplishes data collection, data calculation, and data communication as well as data storage and achieves an organic unification between information of the physical surroundings [1]. The development of the information technology has enabled the appliance of WSNs to various areas like national defense, environment monitoring, rescuing, smart home, e-health, agricultural production, and transportation [2].

Network energy consumption and the coverage quality are two crucial problems for the research of WSNs [3]. The coverage quality determines the monitoring effectiveness for the mobile target. The behavioral characteristic of the coverage quality mainly manifests in the node deployment.
The key to the network energy consumption problem is to effectively control the rapid energy exhaustion, extend the entire network lifetime, and enhance the quality of service (QoS). The network energy efficiency and coverage quality are mainly determined by the reasonability of the deployment of the sensor nodes [4]. Normally, restrained by environmental factors such as the landscape, the sensor nodes are deployed in a random way. No a priori information on the geological information of nodes is available because of the randomness. Meanwhile, multiple sensor nodes may exist within a certain monitoring area or monitoring spot; that is, $k$-coverage comes into existence. In another case, the randomness of deployment may also lead to uncovered monitoring areas or monitoring spots, that is, coverage dead zones which can only be eliminated by introducing more deployed sensor nodes. Coverage is guaranteed for both cases described above. However, some disadvantages inevitably 
exist. Firstly, the high-density clustering of a massive number of sensor nodes would cause a large amount of redundant information and the congestion of the communication link, which restrains the scalability of the network, undermines the network QoS, and shortens the network lifetime. Secondly, real-time monitoring and coverage for the target node concerned cannot be guaranteed when it is located in the coverage dead zone. As a result, the sensor nodes fail to transmit accurate data information to aggregation nodes, which further leads to bias and uncertainty in the collected data information of the aggregation node. Furthermore, the sensor nodes exhibit heterogeneity after several operation cycles, which cause the changes of the node coverage area. Once the target node concerned is no longer covered, the data information collected for the target node will become meaningless. Finally, the nature of coverage is the effective coverage for the target node concerned, instead of all the target nodes. The coverage effectiveness is elaborated as follows. As the target node concerned moves through the area, head nodes accomplish the coverage while its joint nodes are awakened to achieve joint coverage and transmit the collected date information on the mobile target node to the aggregation node. For a deeper understanding of the problem described above, a fusion coverage algorithm based on effective threshold is proposed which could maximize the coverage area with the least sensor nodes and ensure the realtime coverage for the target node concerned.

Based on the discussion above, we perform investigations on the two key problems of the sensor network, that is, the coverage problem and the network lifetime. However, there are still some deviations between the theoretical results and the realistic engineering results for the coverage quality and the network lifetime. Therefore, accurately locating the target nodes, enhancing the coverage intensity for a certain target node, and improving the coverage quality for the target node concerned utilizing fusion coverage are still three open problems which remain to be solved.

To solve these problems, we employ the membership function of coverage intensity and the prediction model for the fusion operator. In order to have a more accurate estimation for the network coverage quality and extend the system lifetime, the analyses are then performed on the range for the effective threshold of the membership function and the maximization problem for the effective coverage intensity as well as the tunable range for the dynamic parameters. Meanwhile, the derivation of the maximal fusion coverage intensity is elaborated utilizing a processing function on the distances from the target node to the ones in the sensor node set. Generally speaking, the proposed algorithm is enlightening for improving energy efficiency for sensor nodes and extending the network lifetime.

\section{Related Works}

The coverage for the target node is accomplished by the cooperation of many sensor nodes. Many experiments have proven that as the target node enters the monitoring area, sensor nodes could sense the target node and then accomplish the full coverage for the target node. However, effective coverage, instead of full coverage, is sufficient for practical applications. The reason is that full coverage is accomplished at the cost of too much network energy consumption which accelerates the network collapse. Recently, the coverage problem of the sensor network has inspired much interest. An ID authentication based optimized coverage protocol was proposed in [5] where the coverage over multiple target nodes is accomplished by different IDs. Meanwhile, the derivation for the coverage quality was presented when multiple target nodes are covered. The different coverage angles of the sensor nodes were exploited by [6] to derive the required area for the coverage sector. Then the coverage quality for the target node from a certain angle can be obtained. A coverage algorithm for multiple targets was presented in [7] based on linear programming and the clustering structure. The optimized coverage over multiple targets is achieved via the calculations of the network coverage probability and the remaining energy for the nodes. The artificial intelligence algorithm was employed in [8] during the coverage process of the sensor nodes, that is, the swarm intelligence algorithm. The complete deployment of the sensor nodes in the entire network monitoring area is accomplished. For the optimization period of the coverage probability, these two intelligent algorithms are employed iteratively for the optimization of the network coverage probability refinement function. Finally, the complete coverage over the monitoring area is achieved. A coverage control algorithm was proposed based on node scheduling strategies [9]. The network coverage quality to demand coverage quality ratio is taken as the indicator of the network performance. Exploiting this relationship, a scheduling strategy was established for the sensor nodes. As a result, the connectivity and coverage over the entire network can be guaranteed with the minimal number of nodes. An enhanced coverage control algorithm (ECCA) was proposed in [10]. The derivation for the expectation of the coverage quality was elaborated when the coverage over the monitoring area was guaranteed. The functional relationships among different parameters were also verified when the random variables were mutually independent. It was also proven that the coverage over the monitoring area can be achieved effectively by adjusting the tunable parameters. The mobility characteristic of the sensor nodes was utilized by [11] to achieve the coverage over the target node, assuming that the network connectivity is guaranteed. Two heuristic algorithms were proposed in [11] where the Voronoi diagram was adopted as the model for the active area of the target nodes. The energy consumption for the sensor nodes is then reduced to accomplish the network energy balance and further improve the network coverage probability. A fence coverage algorithm was proposed in [12] to maximize the network lifetime. This algorithm starts once the moving target node crosses the monitoring area. The correlation between the neighbors and the sensor node is employed to achieve the consistent coverage on the mobile target node. Finally, the complete coverage over the target is achieved. A distributed connectivity and coverage maintenance algorithm was proposed in [13]. In this algorithm, 
the redundant nodes are awakened by the working nodes and the network connectivity is therefore guaranteed. The effective coverage over the target node is maintained with only a small number of mobile sensor nodes. Therefore, the energy consumption caused by the long-distance monitoring can be controlled and the network lifetime can be prolonged. An energy-efficient target coverage algorithm was proposed in [14], which constructs the network clusters based on nonlinear programming. The energy evaluation is performed for each cluster node. The sensor nodes satisfying the coverage condition are chosen as elements of the optimal coverage set. Therefore, the coverage effect is optimized and the network lifetime is prolonged. A probability-driven mechanism based coverage algorithm was proposed in [15]. This algorithm first establishes the coverage model for the sensor nodes and the target nodes. Then the coverage ratio between the sensor nodes and the target nodes is calculated based on probability theory. Finally, the state transitioning process for the nodes is accomplished according to the node scheduling algorithm. As a result, the network lifetime is prolonged.

All the algorithms stated above could accomplish the effective coverage over the monitoring area under some particular conditions. However, they exhibit some disadvantages. For example, the algorithms in [5-7] are high in terms of computational complexity. The maximization of the network lifetime was not considered in [8-10]. The refinement functions in [11-13] vary significantly, which leads to inaccurate calculation results, while the models in $[14,15]$ are too ideal for practical engineering applications. Therefore, we employ the sensing probability model and propose a fusion coverage algorithm based on controllable effective threshold. This algorithm achieves the network fusion coverage by adjusting the tunable threshold parameter and efficiently prolongs the system lifetime.

The structure of this paper is described as follows. Section 3 performs a theoretical analysis on the network model and presents related definitions and the energy decay function for the sensor nodes. The CETFC algorithm is elaborated [16]. Furthermore, the membership function is employed to indicate the coverage intensity. For a further improvement on the coverage quality for the target node concerned, the coverage over the monitoring area is achieved by adjusting the effective threshold. The functional relationship for the distances between the nodes in the sensor node set and the target node is employed to derive the maximum of the fusion coverage intensity. We then display numerical simulation results in Section 4. Compared with other algorithms, the proposed CETFC algorithm exhibits prominent effectiveness and feasibility. Finally, we conclude this paper in Section 5.

\section{Methodology}

3.1. Basic Definitions. For a better study on the coverage problem, we make simplifications by introducing the five following assumptions [10].

Assumption 1. At the initial phase, the sensing ranges and communication ranges for all the nodes are in the shape of a circle while the communication radius is twice the value of the sensing radius.

Assumption 2. The sensor node set lies within the monitoring area. All the nodes are synchronized during the working phase. The boundary effect can be neglected.

Assumption 3. The location information for each node can be obtained via the positioning algorithms while no extra positioning device is needed.

Assumption 4. At the initial phase, all sensor nodes possess the same sensing coverage intensity membership function.

Assumption 5. The sensing area of the sensor nodes is far smaller than the monitoring area.

Definition 6 (coverage intensity). Assuming that the location of an arbitrary sensor node $s_{i}$ is $\left(x_{s}, y_{s}\right)$ while that of the target node $t_{i}$ is $\left(x_{t}, y_{t}\right)$, then the coverage intensity from $s_{i}$ to $t_{i}$ is defined as

$$
I\left(s_{i}, t_{i}\right)= \begin{cases}0 & d\left(s_{i}, t_{i}\right) \geq r+r_{m} \\ e^{-\alpha\left[d\left(s_{i}, t_{i}\right)-r\right]^{\beta}} & r<d\left(s_{i}, t_{i}\right)<r+r_{m} \\ 1 & d\left(s_{i}, t_{i}\right) \leq r,\end{cases}
$$

where $\alpha$ and $\beta$ are physical parameters with $\alpha \in(0,+\infty)$ and $\beta \in(0,1], r$ is the sensing radius for the sensor node after decay, $r_{m}$ is the decay distance for the sensor nodes, $r+r_{m}$ is the sensing radius of the sensor nodes, and $d\left(s_{i}, t_{i}\right)$ indicates the Euclidean distance from the target node to the sensor node.

Definition 7 (effective coverage intensity). Assuming that the effective threshold is $e_{\text {th }}$ with $e_{\text {th }} \in[0,1]$, when the coverage intensity exceeds $e_{\mathrm{th}}$, that is, $I\left(s_{i}, t_{i}\right)>e_{\mathrm{th}}$, the coverage intensity $I\left(s_{i}, t_{i}\right)$ is defined as effective coverage intensity and denoted as $I_{e}\left(s_{i}, t_{i}\right)$. Note that the effective threshold $e_{\text {th }}=$ $\exp (-\alpha r)^{\beta}$ if and only if $d\left(s_{i}, t_{i}\right)=0$.

Definition 8 (membership function). The function employed to indicate the effective coverage intensity is defined as the effective coverage membership function and denoted as $u(x, y)$. The range of the membership function is $[0,1]$. The physical meaning of the membership function is the membership degree of the target node with respect to the coverage from the sensor node. A larger membership function indicates a higher membership degree, and vice versa.

Definition $9\left(\left(M, C_{\mathrm{th}}\right)\right.$ fusion coverage $)$. The fusion coverage intensity from the nodes in the sensor node set $M$ to an arbitrary target node within the monitoring area is denoted as $I_{M}$. Assuming that the effective coverage threshold is $C_{\mathrm{th}}$ with $C_{\mathrm{th}} \in\left(e_{\mathrm{th}}, 1\right]$ and $I_{M} \geq C_{\mathrm{th}}$, then the sensor node set $M$ fusion covers the target node, which is denoted as $\left(M, C_{\mathrm{th}}\right)$. Note that the sensor node set $M$ is composed of $m$ sensor nodes. 
The signal is transmitted over the air and received at the sensor nodes $[17,18]$ within the monitoring area. During the transmission, the signal is attenuated by environmental factors such as physical obstacles, which would lead to uncertainty for the received signal at the sensor nodes [1921]. Furthermore, this will cause errors in the calculation results. Affected by the factors above, the sensing radius of the sensing node mainly depends on the environmental factors in the monitoring area $[22,23]$. During the coverage process from the sensor node to the target node, the loss of energy due to distance is expressed in the exponential form.

$$
P_{L}(d)=P_{L}\left(d_{0}\right)+10 n \log _{10}\left(\frac{d}{d_{0}}\right)+N_{\sigma},
$$

where $d$ indicates the Euclidean distance between the sensor node and the target node, $d_{0}$ is the reference range physical parameter, $n$ stands for the energy decay parameter due to the link distance between the sensor node and the target node, $N_{\sigma}$ is a random variable with mean 0 and variance $\sigma^{2}$, and $P_{L}\left(d_{0}\right)$ is the function for the average decay loss with reference point $d_{0}$, while $P_{L}\left(d_{0}\right)$ is the average decay loss function with reference point $d$. follows:

The received signal at the sensor node can be modelled as

$$
P_{r}=P_{t}-P_{L}\left(d_{0}\right)-10 n \log _{10}\left(\frac{d}{d_{0}}\right)-N_{\sigma},
$$

where $P_{r}$ is the power of the received signal and $P_{t}$ is the transmission power. Due to the impacts of the environmental factors, the power of received signals from different receiving angles is different, even for the sensor nodes at the same location.

3.2. Fusion Coverage with Restricted Threshold. The sensor node set composed of $m$ sensor nodes is denoted as $M$; that is, $M=\left\{s_{1}, s_{2}, s_{3} \ldots, s_{m}\right\}$. For an arbitrary target node $t$ within the monitoring area, the coverage intensity for the target node $t$ is $I\left(s_{i}, t\right)$. According to Definition 9, the fusion coverage intensity of the set $M$ at node $t$ is $I_{M}$. Define the fusion operator as $F:[0,1]^{M} \rightarrow[0,1]$. Then the effective fusion coverage intensity is

$$
\begin{aligned}
I_{M} & =F\left[I\left(s_{1}, t\right), I\left(s_{2}, t\right), \ldots, I\left(s_{m}, t\right)\right] \\
& =\min \left(1, \frac{1}{\lambda}\left(\prod_{i=1}^{M}\left[1+\lambda I\left(s_{i}, t\right)\right]-1\right)\right),
\end{aligned}
$$

in which $\lambda$ is a ratio with range $\lambda \in[-1,0)$. When the fusion coverage intensity from the set $M$ to the target node is no smaller than $C_{\mathrm{th}}$, the target node is $\left(M, C_{\mathrm{th}}\right)$ covered. When the coverage intensity diminishes, the value of $\lambda$ can be adjusted to improve the coverage intensity and achieve the effective coverage over the target node [3].

According to (4), $k$ nodes within the set $M$ could be found out to achieve the maximal fusion coverage intensity as follows:

$$
I_{k}(M, t)=\max _{\left\{s_{1}, s_{2} \cdots s_{k}\right\} \in M} F\left[I\left(s_{1}, t\right), I\left(s_{2}, t\right) \cdots I\left(s_{k}, t\right)\right] .
$$

According to (5), during the coverage from the set $M$ to the target node set $T$, the minimal fusion coverage intensity is

$$
\begin{aligned}
& I_{k_{-} T}(M, T) \\
& \quad=\min _{t \in T}\left\{\max _{\left\{s_{1}, s_{2} \cdots s_{k}\right\} \in M} F\left[I\left(s_{1}, t\right), I\left(s_{2}, t\right) \cdots I\left(s_{k}, t\right)\right]\right\} .
\end{aligned}
$$

Theorem 10. When $m$ equidistant sensor nodes perform the fusion coverage for the target node, the distance $d$ from the sensor node to the target node should satisfy $d \leq r+(-1 /$ $\left.\lambda \ln \left(C_{\mathrm{th}} / \sqrt[q]{m}\right)\right)^{1 / \beta}$, where $q$ is the fusion factor with $q \geq 2$.

Proof. When $m$ equidistant sensor nodes perform the fusion coverage for the target node, the effective fusion coverage intensity should satisfy

$$
I_{m}=F\left(I\left(s_{1}, t\right), I\left(s_{2}, t\right), I\left(s_{3}, t\right) \cdots I\left(s_{m}, t\right)\right) \geq C_{\text {th }} .
$$

According to (4),

$$
I_{m}=\min \left[1, \sum_{i=1}^{m} I\left(s_{i}, t\right)^{q}\right]^{1 / q} \geq C_{\mathrm{th}} .
$$

Since the $m$ sensor nodes are equidistant, that is,

$$
I\left(s_{1}, t\right), I\left(s_{2}, t\right), I\left(s_{3}, t\right) \cdots I\left(s_{m}, t\right)=I_{m},
$$

substituting (9) into (8), we have

$$
\frac{C_{\mathrm{th}}}{\sqrt[q]{m}} \leq I_{m}
$$

According to Definition 6, when $r<d<r+r_{m}$, the value of $I_{m}$ is

$$
I_{m}=\exp \left\{-\lambda[d(s, t)-r]^{-\beta}\right\} .
$$

Substituting (11) into (10), we have

$$
I_{m}=\exp \left\{-\lambda[d(s, t)-r]^{\beta}\right\} \geq \frac{C_{\text {th }}}{\sqrt[q]{m}} .
$$

We further simplify (12):

$$
d \leq r+\left(\frac{-1}{\lambda \ln \left(C_{\mathrm{th}} / \sqrt[q]{m}\right)}\right)^{1 / \beta} .
$$

Therefore, the proof is completed.

Corollary 11. When $m$ equidistant sensor nodes perform the effective coverage for the target node, the critical distance for the effective coverage intensity is

$$
d=r+\left(\frac{-1}{\lambda \ln \left(C_{\mathrm{th}} / \sqrt[q]{m}\right)}\right)^{1 / \beta} .
$$

Proof. According to Theorem 10, the coverage from the equidistant sensor nodes is equivalent. The same is true for the effective coverage intensity. In other words, $I\left(s_{1}, t\right)=$ 
TABle 1: Parameters for simulation.

\begin{tabular}{lc}
\hline Parameter & Value \\
\hline Area I & $100 * 100$ \\
Area II & $200 * 200$ \\
Area III & $300 * 300$ \\
$r$ & $5 \mathrm{~m}$ \\
Time & $100 \mathrm{~s}$ \\
$R$ & $10 \mathrm{~m}$ \\
$E_{R \text {-elec }}$ & $50 \mathrm{~J} / \mathrm{b}$ \\
$E_{T \text {-elec }}$ & $50 \mathrm{~J} / \mathrm{b}$ \\
$\varepsilon_{\mathrm{fs}}$ & $10(\mathrm{~J} / \mathrm{b}) / \mathrm{m}^{2}$ \\
Energy & $2 \mathrm{~J}$ \\
\hline
\end{tabular}

$I\left(s_{2}, t\right)=\cdots=I\left(s_{m}, t\right)=I_{m}$. For the set composed of $m$ sensor nodes, the equivalent distance is

$$
d=r+\left(\frac{-1}{\lambda \ln \left(C_{\mathrm{th}} / \sqrt[q]{m}\right)}\right)^{1 / \beta}
$$

This is exactly the critical distance for an arbitrary sensor node. Therefore, the proof is completed.

\section{Experimental Result}

The energy loss at the transmitting end is modeled as follows:

$$
\begin{aligned}
E_{\mathrm{Tx}}(l, d) & =\mathrm{lE}_{T-\text { elec }}+E_{\mathrm{amp}}(l, d) \\
& = \begin{cases}l \mathrm{E}_{T \text {-elec }}+l \varepsilon_{\mathrm{fs}} d^{2}, & d<d_{0} \\
1 \mathrm{E}_{T \text {-elec }}+l \varepsilon_{\mathrm{amp}} d^{4}, & d \geq d_{0} .\end{cases}
\end{aligned}
$$

The energy loss model for the receiving end is

$$
E_{\mathrm{Rx}}(l)=E_{R \text {-elec }}(l)=1 \mathrm{E}_{\text {elec }},
$$

in which $E_{T \text {-elec }}$ and $E_{R \text {-elec }}$ are the energy consumption for transmitting and receiving 1-bit data, respectively, $d_{0}$ is the Euclidean distance threshold from the node to its communication neighbors, $\varepsilon_{\mathrm{amp}}$ stands for the energy consumption parameter for multipath loss, and $\varepsilon_{\mathrm{fs}}$ is the energy consumption for the node in the free space. When the node performs transmission, the path loss indices are 2 and 4, respectively. Related simulation parameters are elaborated in Table 1.

We make some comparisons, under different algorithm operating time, between the demand coverage probability and the calculated coverage probability. The impact of the dynamic parameter $\lambda$ is also investigated while the simulation area has the size $100 * 100 \mathrm{~m}^{2}$ and the results are obtained by averaging 300 repeated simulations. The simulations are depicted in Figures 1 and 2.

Figures 2 and 3 show the relationship between the demand coverage probability and the network coverage probability for different $\lambda$ and different time. Observe from Figures 2 and 3 that the network coverage probability gets improved with the increasing $\lambda$. The reason is that when

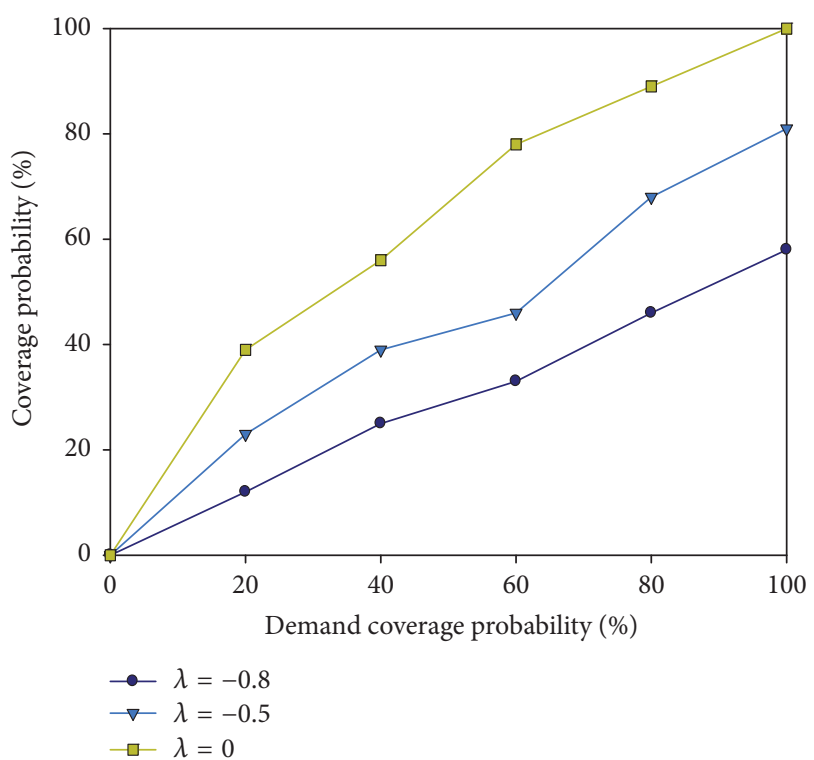

FIGURE 1: The demand coverage probability versus the calculated results when $t=100 \mathrm{~s}$.

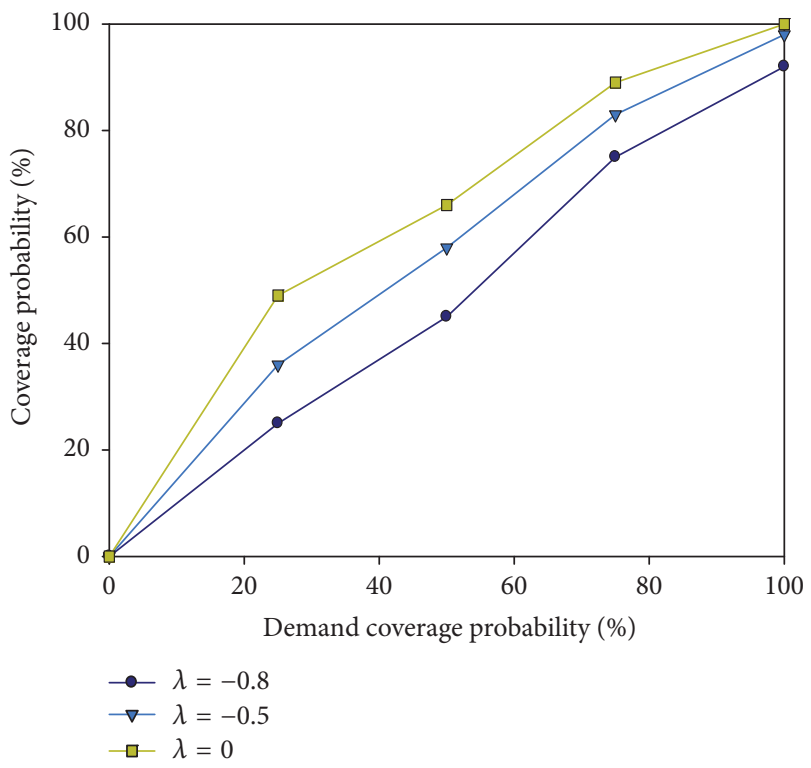

FIGURE 2: The demand coverage probability versus the calculated coverage probability when $t=200 \mathrm{~s}$.

$\lambda$ increases, the effective fusion coverage intensity gets strengthened. When $\lambda=0$, the effective fusion coverage intensity is 1 which is the maximal value. The network coverage probability increases with time in Figures 2 and 3. The reason is that there are more nodes in the sensor in the set $M$; that is, the effective fusion coverage intensity is increased for the target nodes in the monitoring area.

Then, we compare the CETFC algorithm with the ECTA algorithm [13] on network lifetime and operating time.

Figures 3 and 4 show the comparison between our proposed algorithm and the ECTA algorithm on network lifetime and operating time. It can be observed from Figure 3 


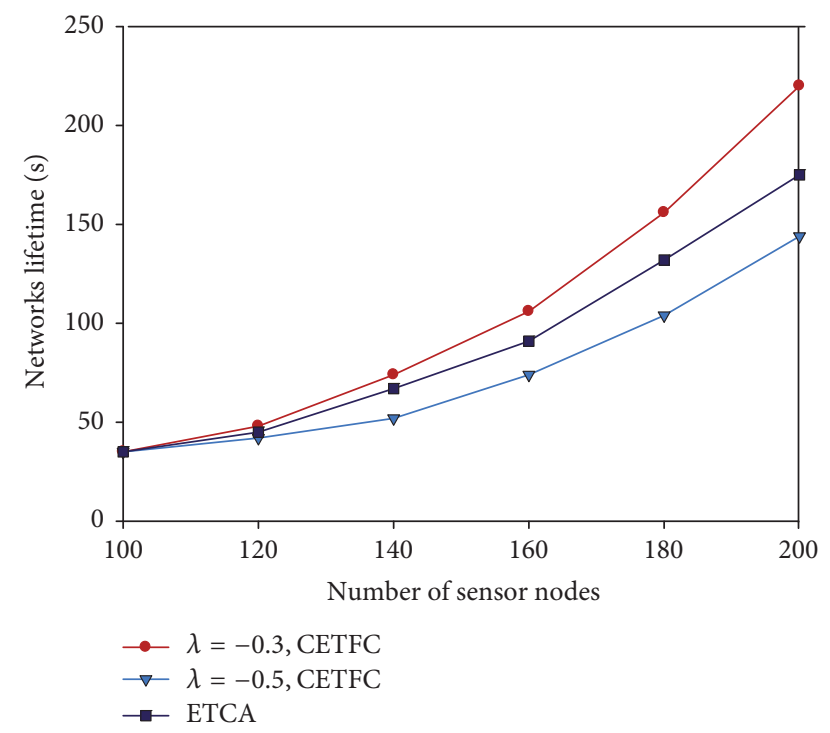

Figure 3: Comparison of network lifetime in monitoring area 200 $* 200 \mathrm{~m}^{2}$.

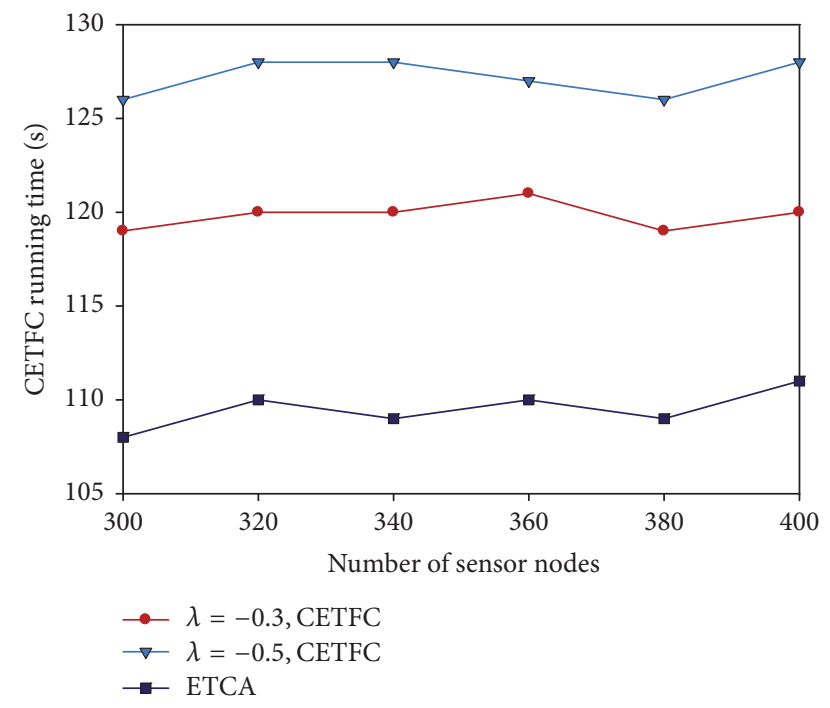

FIgURE 4: Comparison of operation time.

that, at the initial phase, these two algorithms exhibit similar network lifetime which can be further extended for both algorithms with a larger number of sensor nodes. However, since the ECTA algorithm achieves monitoring over the target nodes in a nonlinear and consistent way, more energy consumption is required by the ECTA algorithm. When there are more than 180 sensor nodes, the network lifetime of these two algorithms tends to be stabilized. The average system lifetime of the CETFC algorithm is higher than that of the ECTA algorithm by $12.78 \%$. The algorithm operating time is presented in Figure 4 as a function of sensor node number. The ECTA algorithm employs a link structure for the storage of node energy. The node energy is ranked by an ergodic algorithm and the node with more energy is given higher priority to achieve the coverage over the target node. As a

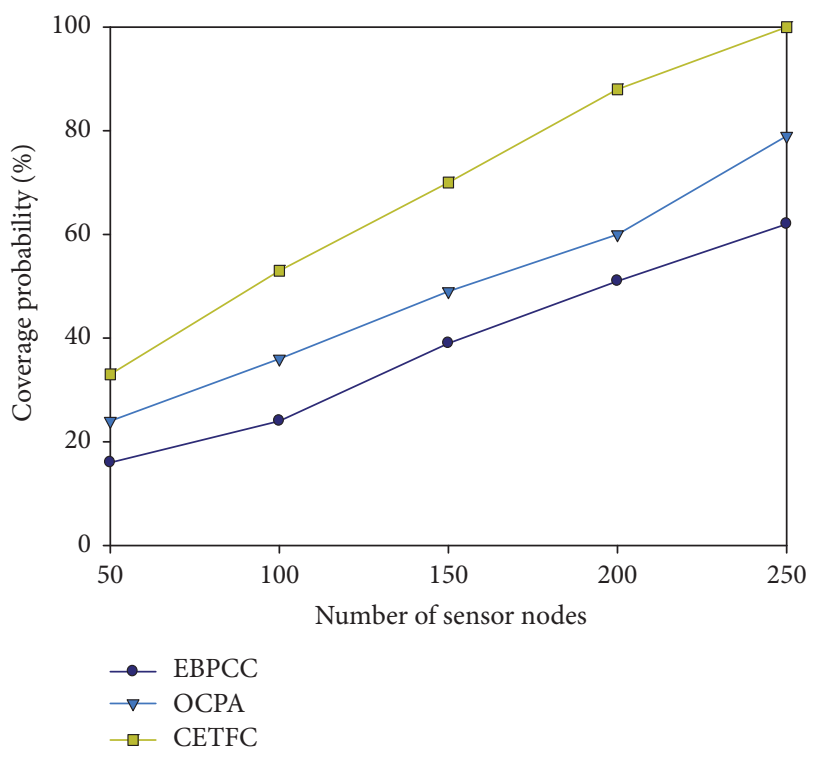

FIGURE 5: Comparison of network coverage probability for the three algorithms in simulation area $200 * 200 \mathrm{~m}^{2}$.

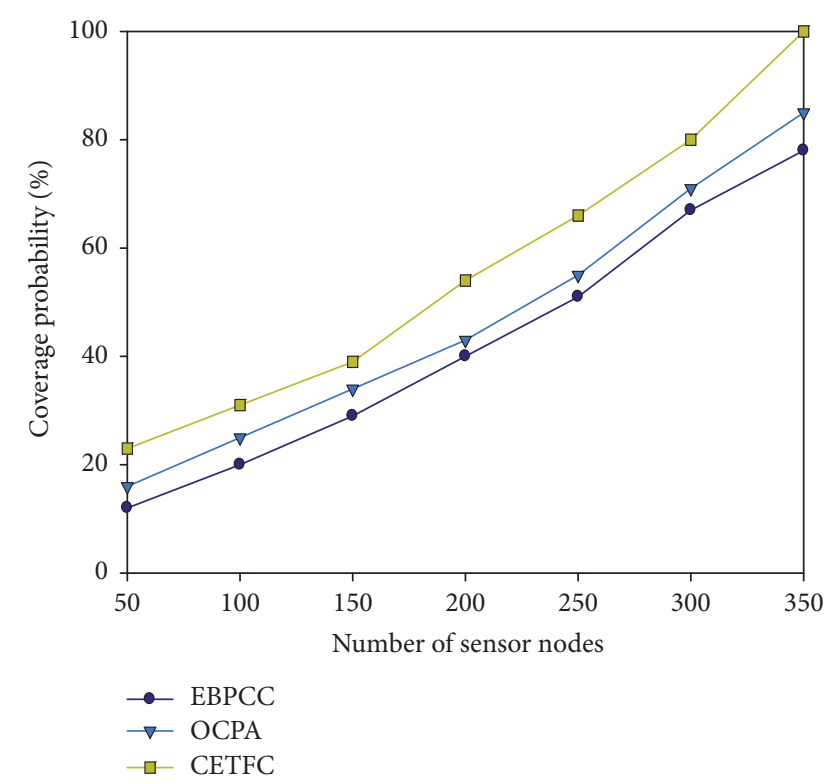

FIGURE 6: Comparison of network coverage probability for the three algorithms in simulation area $200 * 200 \mathrm{~m}^{2}$.

result, the ECTA algorithm possesses lower complexity and requires less operating time in comparison with our proposed algorithm.

We compare our proposed algorithm with the algorithms in $[2,9]$ in terms of network coverage probability and network lifetime. The simulation areas are $200 * 200 \mathrm{~m}^{2}$ and $300 *$ $300 \mathrm{~m}^{2}$, respectively, and the operating time is $t=200 \mathrm{~s}$. The results are obtained by averaging 300 simulations. Simulation results are illustrated in Figures 5-7.

It is clearly evident from Figures 5 and 6 that the coverage probability gets improved for all of the three algorithms when there are more sensor nodes. However, our proposed 


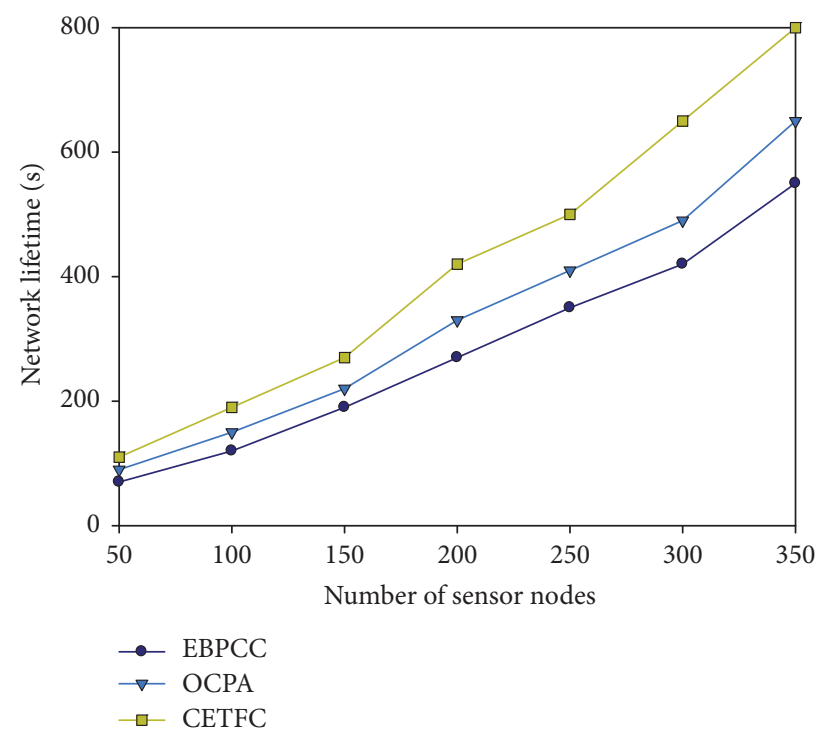

FIgURE 7: Comparison of network lifetime for the three algorithms in simulation area $200 * 200 \mathrm{~m}^{2}$.

algorithm shows a higher improvement speed than the others. This is due to the fact that the CETFC algorithm adopts the concept of fusion coverage intensity where the coverage intensity of all the nodes in the set $M$ is taken into consideration. As a result, the effective fusion coverage intensity from the entire set $M$ to the target node gets further improved. When there are equal numbers of sensor nodes, the proposed algorithm shows higher network coverage probability than any of the other algorithms while the average improvement rate is $10.12 \%$. Observe from Figure 7 that, with the same number of sensor nodes, the proposed algorithm shows longer network lifetime than the other two algorithms and the average improvement rate is $13.68 \%$. The reason behind the improvement on the network lifetime is the same as that for the improvement on the network coverage probability.

\section{Conclusions}

For a better investigation on the coverage problem in WSNs, we employed the probability theory to investigate the network coverage quality and the network lifetime. We revealed the proportional relationship between the fusion coverage intensity and the membership function during the coverage over the target node. We also investigated the range of the dynamic parameter $\lambda$ and the fusion coverage process over the target node set. Finally, we obtained the following conclusions.

(1) The energy of the sensor node decays exponentially and exhibits a reverse proportional relationship with the Euclidean distance.

(2) The restricted threshold is employed to indicate the fusion coverage intensity and provide an accurate calculation for the range of the fusion coverage intensity. As a result, the effective coverage process from the sensor nodes to the target node can be reflected effectively.
(3) By setting the effective threshold, the coverage intensity can by adjusted to optimize the network resource allocation, reduce the network cost, improve the network energy efficiency, and prolong the network lifetime.

(4) By adjusting the tunable parameters, the fusion coverage intensity can be increased effectively to improve the coverage quality over the monitoring area.

We focused on the probability model and proposed a controllable effective threshold based fusion coverage algorithm. The node energy decay model was further simplified for computational considerations. The CETFC algorithm shows certain reference value to subsequent study on the network coverage quality. Since the study has been performed theoretically so far, it requires future work to put this algorithm into practice and improve the network coverage quality in engineering applications.

\section{Conflicts of Interest}

The authors declare that they do not have any commercial or associative interests that represent conflicts of interest in connection with the work submitted.

\section{Acknowledgments}

This work is supported by the project of the First-Class University and the First-Class Discipline (no. 10301-017 004011501) and the National Natural Science Foundation of China.

\section{References}

[1] A. E. Zonouz, L. Xing, V. M. Vokkarane, and Y. Sun, "Hybrid wireless sensor networks: A reliability, cost and energy-aware approach," IET Wireless Sensor Systems, vol. 6, no. 2, pp. 42-48, 2016.

[2] R. V. Kulkarni and G. K. Venayagamoorthy, "Particle swarm optimization in wireless-sensor networks: a brief survey," IEEE Transactions on Systems, Man, and Cybernetics, Part C: Applications and Reviews, vol. 41, no. 2, pp. 262-267, 2011.

[3] Z. Sun, Y. Zhang, Y. Nie, W. Wei, J. Lloret, and H. Song, "CASMOC: a novel complex alliance strategy with multiobjective optimization of coverage in wireless sensor networks," Wireless Networks, 2016.

[4] J.-T. Meng, J.-R. Yuan, S.-Z. Feng, and Y.-J. Wei, "An energy efficient clustering scheme for data aggregation in wireless sensor networks," Journal of Computer Science and Technology, vol. 28, no. 3, pp. 564-573, 2013.

[5] H. Mahboubi, K. Moezzi, A. G. Aghdam, K. Sayrafian-Pour, and V. Marbukh, "Distributed deployment algorithms for improved coverage in a network of wireless mobile sensors," IEEE Transactions on Industrial Informatics, vol. 10, no. 1, pp. 163-175, 2014.

[6] Y.-C. Tseng, P.-Y. Chen, and W.-T. Chen, "K-angle object coverage problem in a wireless sensor network," IEEE Sensors Journal, vol. 12, no. 12, pp. 3408-3416, 2012.

[7] N. Tamboli and M. Younis, "Coverage-aware connectivity restoration in mobile sensor networks," Journal of Network and Computer Applications, vol. 33, no. 4, pp. 363-374, 2010. 
[8] C.-C. Hsu, M.-S. Kuo, S.-C. Wang, and C.-F. Chou, "Joint design of asynchronous sleep-wake scheduling and opportunistic routing in wireless sensor networks," Institute of Electrical and Electronics Engineers. Transactions on Computers, vol. 63, no. 7, pp. 1840-1846, 2014.

[9] F. Lin, X. Z. Zhou, and W. H. Zeng, "Sparse online learning for collaborative filtering," International Journal of Computers Communications \& Control, vol. 11, no. 2, 2016.

[10] M. R. Senouci, A. Mellouk, L. Oukhellou, and A. Aissani, "An evidence-based sensor coverage model," IEEE Communications Letters, vol. 16, no. 9, pp. 1462-1465, 2012.

[11] F. Lin, W. Zeng, L. Yang, Y. Wang, S. Lin, and J. Zeng, "Cloud computing system risk estimation and service selection approach based on cloud focus theory," Neural Computing and Applications, vol. 28, no. 7, pp. 1863-1876, 2017.

[12] J.-H. Seok, J.-Y. Lee, W. Kim, and J.-J. Lee, "A bipopulationbased evolutionary algorithm for solving full area coverage problems," IEEE Sensors Journal, vol. 13, no. 12, pp. 4796-4807, 2013.

[13] X. F. Xing, G. J. Wang, and J. Li, "Polytype target coverage scheme for heterogeneous wireless sensor networks using linear programming," Wireless Communications and Mobile Computing, vol. 14, no. 14, pp. 1397-1408, 2014.

[14] K. Derr and M. Manic, "Wireless sensor network configurationpart II: adaptive coverage for decentralized algorithms," IEEE Transactions on Industrial Informatics, vol. 9, no. 3, pp. 17281738, 2013.

[15] F.-Z. Meng, H.-Z. Wang, and H. He, "Connected coverage protocol using cooperative sensing model for wireless sensor networks," Acta Electronica Sinica, vol. 39, no. 4, pp. 772-779, 2011.

[16] Z. Y. Sun, W. G. Wu, H. Z. Wang, H. Chen, and X. F. Xing, "A novel coverage algorithm based on event-probability-driven mechanism in wireless sensor network," EURASIP Journal on Wireless Communications and Networking, vol. 2014, article 58, pp. 1-17, 2014.

[17] M. Wazid, A. K. Das, S. Kumari, and M. K. Khan, "Design of sinkhole node detection mechanism for hierarchical wireless sensor networks," Security and Communication Networks, vol. 9, no. 17, pp. 4596-4614, 2016.

[18] H. Barati, A. Movaghar, and A. M. Rahmani, "EACHP: Energy Aware Clustering Hierarchy Protocol for Large Scale Wireless Sensor Networks," Wireless Personal Communications, vol. 85, no. 3, pp. 765-789, 2015.

[19] M. Alicherry, R. Bhatia, and L. E. Li, "Joint channel assignment and routing for throughput optimization in multiradio wireless mesh networks," IEEE Journal on Selected Areas in Communications, vol. 24, no. 11, pp. 1960-1971, 2006.

[20] A. Vajdi, G. Zhang, Y. Wang, and T. Wang, "A New SelfManagement Model for Large-Scale Event-Driven Wireless Sensor Networks," IEEE Sensors Journal, vol. 16, no. 20, pp. 7537-7544, 2016.

[21] X. F. Xing, G. J. Wang, and J. Li, "Square region-based coverage and connectivity probability model in WSNs," in Proceedings of the Mobile Ad-Hoc and Sensor Networks 2010 Sixth International Conference (MSN), pp. 79-84, 2011.

[22] Z. Sun, Y. Shu, X. Xing, W. Wei, H. Song, and W. Li, "LPOCS: A novel linear programming optimization coverage scheme in wireless sensor networks," Ad Hoc \& Sensor Wireless Networks, vol. 33, no. 1-4, pp. 173-197, 2016.
[23] C.-L. Yang and K.-W. Chin, "Novel algorithms for complete targets coverage in energy harvesting wireless sensor networks," IEEE Communications Letters, vol. 18, no. 1, pp. 118-121, 2014. 


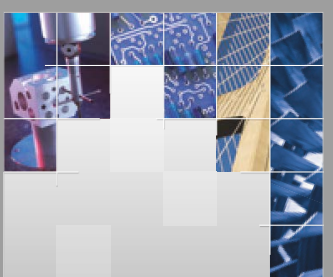

\section{Enfincering}
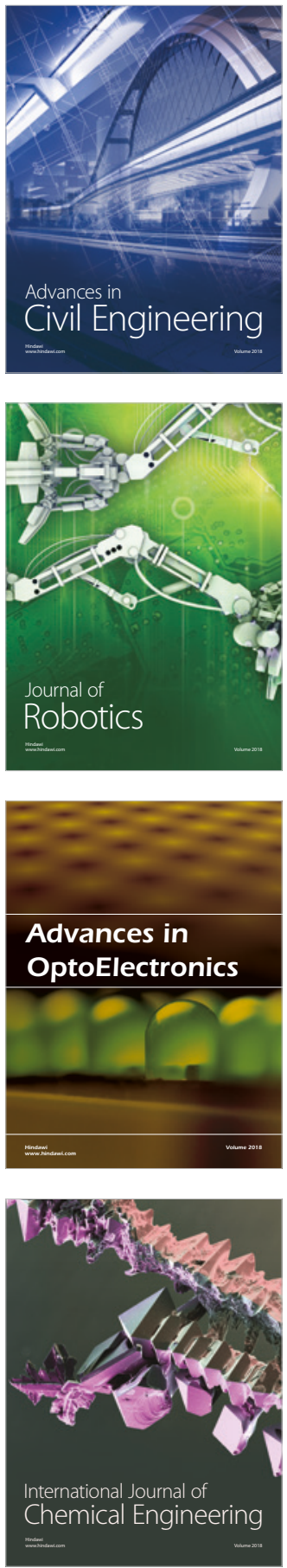

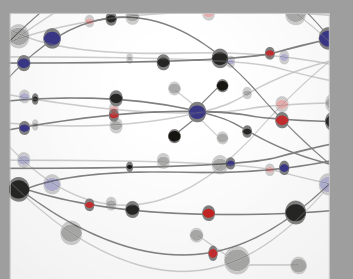

\section{Rotating \\ Machinery}

The Scientific World Journal

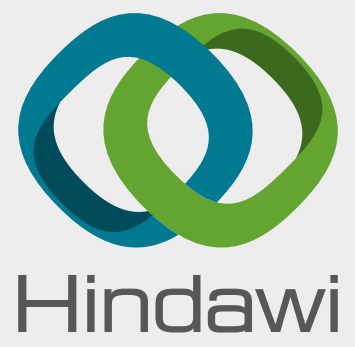

Submit your manuscripts at

www.hindawi.com
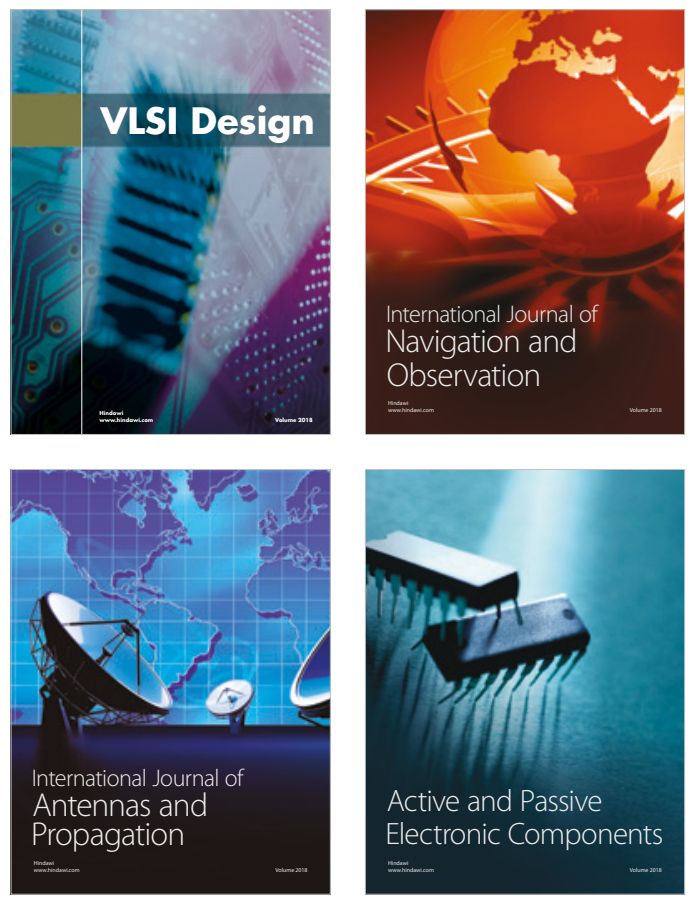
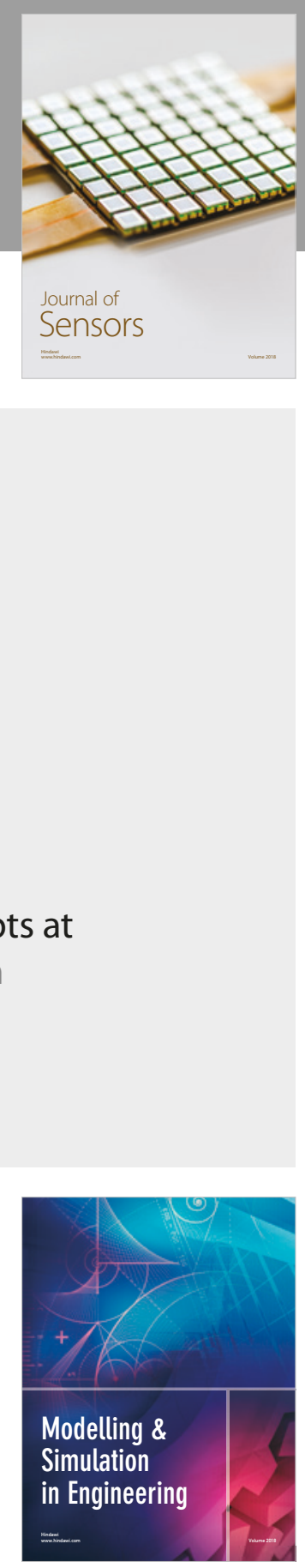

\section{Advances \\ Multimedia}
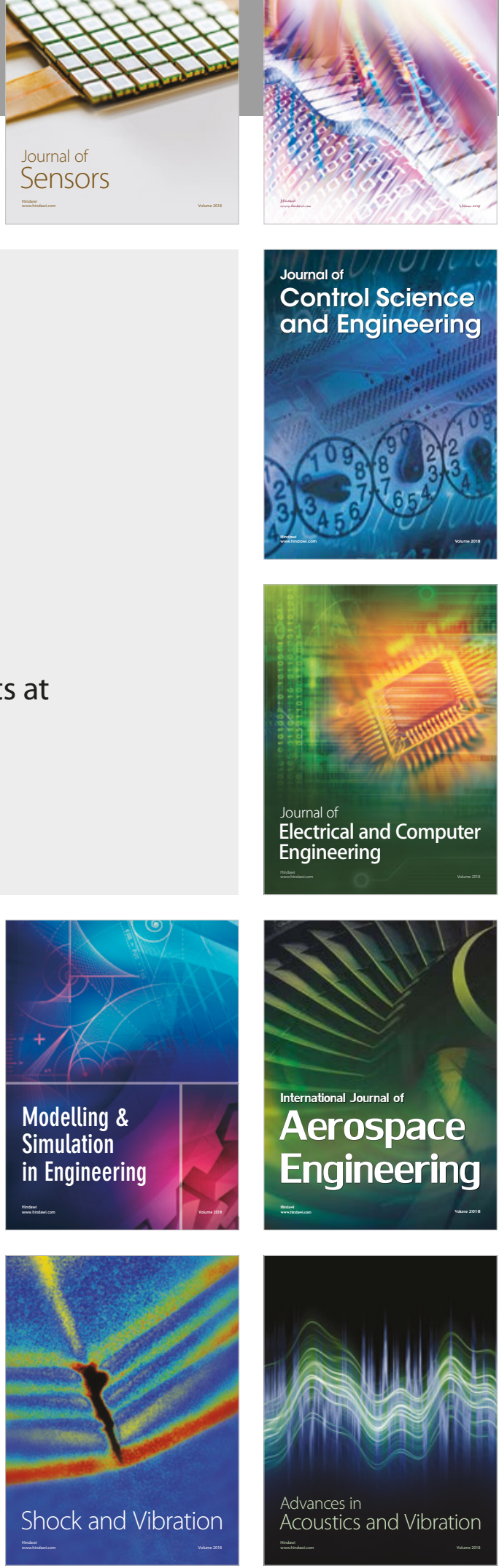